\title{
Gravitational waves from minisplit SUSY
}

\author{
Bartosz Fornal $\odot,{ }^{1}$ Barmak Shams Es Haghi $\odot,{ }^{2}$ Jiang-Hao Yu, ${ }^{3,4,5,6,7}$ and Yue Zhao ${ }^{2}$ \\ ${ }^{1}$ Department of Physical Sciences, Barry University, Miami Shores, Florida 33161, USA \\ ${ }^{2}$ Department of Physics and Astronomy, University of Utah, Salt Lake City, Utah 84112, USA \\ ${ }^{3}$ CAS Key Laboratory of Theoretical Physics, Institute of Theoretical Physics, \\ Chinese Academy of Sciences, Beijing 100190, China \\ ${ }^{4}$ School of Physical Sciences, University of Chinese Academy of Sciences, Beijing 100049, China \\ ${ }^{5}$ Center for High Energy Physics, Peking University, Beijing 100871, China \\ ${ }^{6}$ School of Fundamental Physics and Mathematical Sciences, \\ Hangzhou Institute for Advanced Study, UCAS, Hangzhou 310024, China \\ ${ }^{7}$ International Centre for Theoretical Physics Asia-Pacific, Beijing/Hangzhou 100190, China
}

(Received 9 April 2021; revised 10 October 2021; accepted 8 November 2021; published 6 December 2021)

\begin{abstract}
We show that color-breaking vacua may develop at high temperature in the minisplit supersymmetry (SUSY) scenario. This can lead to a nontrivial cosmological history of the Universe, including strong first order phase transitions and domain wall production. Given the typical PeV energy scale associated with minisplit SUSY models, a stochastic gravitational wave background at frequencies around $1 \mathrm{kHz}$ is expected. We study the potential for detection of such a signal in future gravitational wave experiments.
\end{abstract}

DOI: 10.1103/PhysRevD.104.115005

\section{INTRODUCTION}

The direct detection of propagating gravitational waves (GWs) by LIGO [1] was certainly a milestone discovery. It gave rise to a completely new field, GW astronomy, which is of great importance not only for astrophysics but also high energy physics. One example of how particle physics benefits from this great progress is the opportunities arising from searches for strong first order phase transitions (FOPTs), cosmic strings, and domain walls (DWs) in the early Universe, directly related to the physics at the high scale. A particularly interesting case is when the stochastic GW background is produced by a FOPT at the $\mathcal{O}(\mathrm{PeV})$ scale, since the corresponding signal lies within the sensitivity range of current and future ground-based GW detectors, such as LIGO, Einstein Telescope [2], and Cosmic Explorer [3].

To this day, supersymmetry (SUSY) is one of the most appealing frameworks for physics beyond the Standard Model (SM) and predicts the existence of superpartners of SM particles. The SUSY solution to the gauge hierarchy problem suggests that masses of superparticles should not be too far from the electroweak scale. This is the reason why the Minimal Supersymmetric Standard Model (MSSM) [4-8],

Published by the American Physical Society under the terms of the Creative Commons Attribution 4.0 International license. Further distribution of this work must maintain attribution to the author(s) and the published article's title, journal citation, and DOI. Funded by SCOAP ${ }^{3}$. with superparticles at the $\mathcal{O}(\mathrm{TeV})$ scale, has been thoroughly explored, both experimentally and theoretically. In the MSSM, the charges of superparticles are determined by the quantum numbers of the SM particle content. This imposes strict constraints on the shape of the scalar potential. Detailed studies of the zero temperature and finite temperature vacuum structure of the MSSM with TeV-scale SUSY were carried out in Refs. [9-15]. However, null results in various SUSY searches at the LHC imply that the superparticle masses may actually be at higher energy scales.

Apart from the direct experimental searches indicating that superparticles should be rather heavy, there are also theoretical arguments in support of this scenario. A generic choice of parameters in the MSSM suffers from flavorchanging neutral current (FCNC) and charge-parity violation problems unless the masses of superparticles are at the $\mathcal{O}(100 \mathrm{TeV})$ scale or beyond. In addition, within the MSSM framework, superparticles with large masses can increase the Higgs mass, through quantum corrections, from below the $Z$ boson mass up to $\sim 125 \mathrm{GeV}$, so that it agrees with the value measured at the LHC. A framework with such heavy superparticles is offered by the minisplit SUSY [16-18].

A promising place to look for such high-scale new physics is the early Universe. At temperatures above $\mathcal{O}(100 \mathrm{TeV})$, the scalar fields corresponding to superparticles in the minisplit SUSY become energetically accessible, and their landscape is highly nontrivial. A natural question is whether the existence of these superparticles generically triggers new phenomena in the history of the early Universe that can lead to interesting signatures. 
For example, if the FOPT happened at temperatures $\mathcal{O}(\mathrm{PeV})$, the GW signal would fall within the frequency range accessible by ground-based GW detectors, making them very powerful probes of high energy physics which is not accessible by any other terrestrial experiments, e.g., accelerators like the LHC.

In this study, we demonstrate that a particular realization of the superparticle mass spectrum can lead to an intriguing evolution of the MSSM scalar potential. Specifically 1) at a very high temperature, the global minimum of the scalar potential is situated at the origin of the squark field space; 2) as the temperature decreases, one (or several) of the scalar field directions develops a symmetry-breaking minimum away from the origin, and a FOPT to the new vacuum may happen; and 3) when the temperature drops further, the symmetry-breaking minimum along the squark direction disappears, and the Universe settles into the electroweak symmetry-breaking minimum along the Higgs direction. We show that such an evolution of the Universe leads to the production of GWs, and we discuss its accessibility in future GW detectors.

\section{SYMMETRY NONRESTORATION}

The standard lore is that increasing temperature leads to an enhancement of symmetry. Indeed, for majority of particle physics models, finite temperature effects tend to wash out any nontrivial vacuum structures existing at zero temperature. However, as it was pointed out a long time ago [19], there are cases when increasing temperature actually leads to a broken phase. Since then, many models of this type have been proposed (see, e.g., Refs. [20-38] and references therein).

An example of such a theory is given by the following extension of the SM Lagrangian, obtained by adding one or more scalar fields $s$,

$$
-\mathcal{L}=-\mathcal{L}_{\mathrm{SM}}+\frac{\mu_{s}^{2}}{2} s^{2}+\frac{\lambda_{s}}{4} s^{4}+\frac{\lambda_{h s}}{4} h^{2} s^{2} .
$$

The mixed quartic coupling $\lambda_{h s}$ can be negative, as long as $\lambda_{h s} \geq-\sqrt{\lambda_{s} \lambda}$, where $\lambda$ is the Higgs quartic coupling, so that a negative runaway direction is avoided. In the high temperature limit, the thermal Higgs mass squared is given by

$$
m_{h}^{2}(T) \simeq \frac{1}{2}\left(\frac{\lambda_{t}^{2}}{2}+\lambda+N_{s} \frac{\lambda_{h s}}{6}\right) T^{2},
$$

where $N_{s}$ is the number of fields $s$ added into the theory, $\lambda_{t}$ is the top Yukawa coupling, and we ignored the subleading terms proportional to the electroweak couplings. For a sufficiently negative value of $N_{s} \lambda_{h s}$, the Higgs thermal mass becomes negative, which leads to the development of new minima of the effective potential [33]. Such symmetry nonrestoration and formation of new vacuum states may result in a FOPT at high temperatures, which, in turn, would give rise to $\mathrm{GW}$ signals.

It has been argued that symmetry nonrestoration at high temperature cannot happen in supersymmetric theories [39-41], with the exception of models with flat directions $[42,43]$ or a nonzero background charge [44]. Although these arguments hold for the MSSM at temperatures above the mass scale of all superparticles, they do not apply when the temperature is at intermediate scales. In particular, if there exists a mass separation among the superparticles, which can be realized naturally in various SUSY-breaking scenarios, symmetry nonrestoration can be achieved, within the framework of the minisplit SUSY, at temperatures lower than the mass scale of the heaviest superparticles.

To show this explicitly, let us focus on the $\mathrm{SU}(3)_{c}$ $D$-term contribution to the MSSM scalar potential [45],

$$
V_{D}=\frac{g_{s}^{2}}{6}\left(\sum_{\tilde{q}_{L}}\left|\tilde{q}_{L}\right|^{2}-\sum_{\tilde{q}_{R}}\left|\tilde{q}_{R}\right|^{2}\right)^{2}
$$

where the sum is over the left-handed (LH) and righthanded (RH) squark flavors $\tilde{q}_{L}$ and $\tilde{q}_{R}$. If the squark masses are above the temperature scale, the corresponding thermal corrections to the effective potential are suppressed.

The mass spectrum for superparticles is model dependent. Their interactions, especially the charge assignment under the SM gauge group, may affect the masses. For example, in the framework of gauge mediation, the soft SUSY-breaking masses of superparticles are generated by gauge couplings through a spurious SUSY-breaking sector. We will assume that this or an analogous mechanism makes all LH squarks lighter than the RH squarks.

Furthermore, it is natural to expect that a moderate mass gap appears for squarks of different flavors, similarly to the mass hierarchy for the SM quarks. This can be induced either by a flavor dependence in the SUSY-breaking mediation mechanism, e.g., when the Yukawa couplings are involved, or through renormalization group running from a higher energy scale. Motivated by this, we take the soft mass of $\tilde{d}_{R}$ to be smaller than those of the remaining RH squarks.

With such assumptions regarding the particle spectrum, at temperatures above the mass of $\tilde{d}_{R}$ but below the masses of other RH squarks, the $D$-term contribution to the potential is approximately

$$
V_{D}=\frac{g_{s}^{2}}{6}\left(\sum_{\tilde{q}_{L}}\left|\tilde{q}_{L}\right|^{2}-\left|\tilde{d}_{R}\right|^{2}\right)^{2} .
$$

In the discussion below, we demonstrate that the effective potential of the MSSM with the tree-level contribution in Eq. (4) develops a symmetry-breaking vacuum along the squark $\tilde{d}_{R}$ direction. Depending on the initial conditions for 
the evolution of the early Universe, the existence of this vacuum leads to the possibility of a strong FOPT.

\section{THERMAL EFFECTIVE POTENTIAL}

Without loss of generality, we consider the color direction $(1,0,0)$ and analyze the potential in terms of the field $\tilde{d}_{R 1}$. Upon separating the real and imaginary parts,

$$
\tilde{d}_{R 1}=\frac{1}{\sqrt{2}}\left(\phi_{d}+i a_{d}\right),
$$

the effective potential becomes a function of $\phi_{d}$ and consists of three contributions: tree-level $V_{\text {tree }}\left(\phi_{d}\right)$, oneloop Coleman-Weinberg $V_{\text {loop }}\left(\phi_{d}\right)$, and finite temperature $V_{\text {temp }}\left(\phi_{d}, T\right)$,

$$
V_{\text {eff }}\left(\phi_{d}, T\right)=V_{\text {tree }}+V_{\text {loop }}+V_{\text {temp }} .
$$

The relevant contributions to the tree-level part come from the soft masses and the $\mathrm{SU}(3)_{c} D$-term in Eq. (4),

$$
V_{\text {tree }}=\frac{1}{2} m_{\tilde{d}}^{2} \phi_{d}^{2}+\frac{g_{s}^{2}}{24} \phi_{d}^{4} .
$$

Since we are considering the potential in the direction of the RH squark $\tilde{d}_{R 1}$, the $\mathrm{SU}(2)_{L} D$-term does not contribute to the field-dependent masses. We also neglect the $\mathrm{U}(1)_{Y}$ $D$-term, since its effect is subleading.

Gauginos and Higgsinos are usually assumed to be lighter than squarks in the minisplit scenario, due to $R$ symmetry and Peccei-Quinn symmetry. However, their masses are free parameters and depend on the details of the model. For simplicity, we assume that the gauginos and Higgsinos are much heavier, and the corresponding interactions, such as the gaugino-squark-quark coupling, can be neglected. Denoting by $m_{i}^{2}$ the eigenvalues of the fielddependent squared mass matrix $m_{i, j}^{2}$ given by

$$
\begin{aligned}
m_{i, j}^{2}\left(\phi_{d}\right)= & \left.\frac{1}{2} \frac{\partial^{2} V}{\partial\left[\operatorname{Re}\left(\tilde{q}_{i}\right)\right] \partial\left[\operatorname{Re}\left(\tilde{q}_{j}\right)\right]}\right|_{\operatorname{Re}\left(\tilde{d}_{R 1}\right)=\frac{\phi_{d}}{\sqrt{2}}} \\
& +\left.\frac{1}{2} \frac{\partial^{2} V}{\partial\left[\operatorname{Im}\left(\tilde{q}_{i}\right)\right] \partial\left[\operatorname{Im}\left(\tilde{q}_{j}\right)\right]}\right|_{\operatorname{Im}\left(\tilde{q}_{i}\right)=0},
\end{aligned}
$$

we arrive at the squared masses: $g_{s}^{2} \phi_{d}^{2} / 2$ for $\operatorname{Re}\left(\tilde{d}_{R 1}\right)$, $g_{s}^{2} \phi_{d}^{2} / 6$ for the remaining real and imaginary components of $\tilde{d}_{R}$, and $-g_{s}^{2} \phi_{d}^{2} / 6$ for the LH squark components.

The Coleman-Weinberg contribution is [46]

$$
V_{\text {loop }}=\sum_{i} \frac{n_{i}}{64 \pi^{2}}\left\{m_{i}^{4}\left[\log \left(\frac{m_{i}^{2}}{\mu^{2}}\right)-c_{i}\right]\right\},
$$

where the sum is over all particles in the theory with fielddependent masses, $n_{i}$ is the number of degrees of freedom with an extra minus sign for fermions, $c_{i}=3 / 2$ for scalars and fermions, $c_{i}=5 / 6$ for vector bosons, and $\mu$ is the renormalization scale.

The temperature-dependent part of the effective potential consists of the one-loop finite temperature term and, in case of bosons, the contribution from Daisy diagrams,

$$
V_{\text {temp }}=V_{\text {temp }}^{(1)}+V_{\text {temp }}^{(2)},
$$

where [46]

$$
\begin{aligned}
& V_{\text {temp }}^{(1)}=\frac{T^{4}}{2 \pi^{2}} \sum_{i} n_{i} \int_{0}^{\infty} d y y^{2} \log \left(1 \mp e^{-\sqrt{m_{i}^{2} / T^{2}+y^{2}}}\right), \\
& V_{\text {temp }}^{(2)}=-\frac{T}{12 \pi} \sum_{j} n_{j}^{\prime}\left\{\left[m_{j}^{2}+\Pi_{j}(T)\right]^{\frac{3}{2}}-m_{j}^{3}\right\},
\end{aligned}
$$

with the negative sign for bosons and the positive sign for fermions. The sum over $i$ includes all particles with fielddependent masses, and the sum over $j$ includes only bosons. The squark and gluon thermal masses $\Pi_{j}(T)$ can be calculated following the prescription in Refs. [47,48] and are given by

$$
\Pi_{j}(T)=\frac{4}{9} g_{s}^{2} T^{2}, \quad \Pi_{g}(T)=\frac{19}{6} g_{s}^{2} T^{2} .
$$

We note that some field-dependent squared masses are negative, resulting in the one-loop zero temperature and finite temperature contributions to the potential developing imaginary parts. However, as shown in Ref. [49] (see also Ref. [50]), the imaginary terms cancel between the various contributions (this happens for any $T$, not only at high temperature), and the total effective potential is real.

\section{VACUA IN THE EFFECTIVE POTENTIAL}

Useful intuition can be developed by analyzing the effective potential in the limit

$$
m_{\tilde{d}} \ll T \ll M_{\tilde{q}},
$$

where $m_{\tilde{d}}$ denotes the soft mass of $\tilde{d}_{R}$ (assumed to be small), whereas $M_{\tilde{q}}$ is the soft mass scale for the remaining heavy RH squarks. Denoting collectively $\Pi_{j}(T)=c_{j} T^{2}$, the finite temperature terms can be approximated by

$$
\begin{aligned}
& V_{\text {temp }}^{(1)} \simeq-\frac{\pi^{2}}{90} T^{4}+\frac{T^{2}}{24} \sum_{i} n_{i} m_{i}^{2}, \\
& V_{\text {temp }}^{(2)} \simeq-\frac{T^{4}}{12 \pi} \sum_{i} c_{i}{ }^{3 / 2}-\frac{T^{2}}{8 \pi} \sum_{j} n_{j} \sqrt{c_{j}} m_{j}^{2} .
\end{aligned}
$$

Using Eq. (8), one arrives at 


$$
\sum_{j} n_{j} m_{j}^{2}=-\frac{14}{3} g_{s}^{2} \phi_{d}^{2}
$$

The only other particles developing nonzero fielddependent masses are five of the gluons, each with $n_{g}=3$, yielding

$$
\sum_{g} n_{g} m_{g}^{2}=4 g_{s}^{2} \phi_{d}^{2}
$$

Equations (12), (14), (15), and (16) give the thermal fielddependent contribution to the effective potential equal to

$$
\Delta V_{\mathrm{eff}}=C \phi_{d}^{2} T^{2}
$$

where the constant $C$, upon setting $g_{s}(1 \mathrm{PeV}) \approx 0.8$, is

$$
C \approx-0.003 \text {. }
$$

Since $C<0$, the effective potential receives a negative contribution to the quadratic terms at finite temperature, which leads to the development of a vacuum expectation value (vev) with the size governed by the temperature

$$
v(T) \sim T .
$$

Therefore, in the regime $m_{\tilde{d}} \lesssim T \lesssim M_{\tilde{q}}$, finite temperature effects give rise to symmetry nonrestoration within the framework of the minimal minisplit SUSY itself.

Figure 1 shows the effective potential for several values of the temperature, assuming that the scale of the heavy RH squarks is $M_{\tilde{q}}=10 \mathrm{PeV}$, also taken to be the renormalization scale for the Coleman-Weinberg term. The soft mass for the light RH squark $\tilde{d}_{R}$ is $m_{\tilde{d}}=1 \mathrm{PeV}$. For temperatures $T<1.8 \mathrm{PeV}$, the nontrivial vacuum structure vanishes due to the small size of the thermal contributions in Eq. (17), leaving only one vacuum at $\phi_{d}=0$.

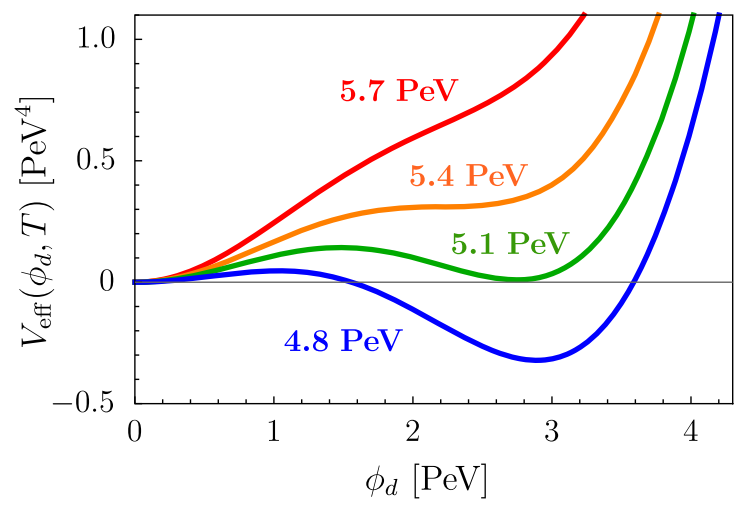

FIG. 1. Effective potential $V_{\text {eff }}\left(\phi_{d}, T\right)$ for four temperatures: 4.8, 5.1, 5.4, and 5.7 PeV, assuming $m_{\tilde{d}}=1 \mathrm{PeV}, M_{\tilde{q}}=10 \mathrm{PeV}$ and upon subtracting off $V_{\text {eff }}(0, T)$. The nontrivial vacuum structure disappears for $T<1.8 \mathrm{PeV}$.
The symmetry is also restored at temperatures $T \gg M_{\tilde{q}}$; thus, the scenario is consistent with the conclusions of Refs. [39-41].

\section{GRAVITATIONAL WAVES}

In this section, we investigate the strength and spectrum of GWs produced by FOPTs in the minisplit SUSY. In our calculation, we use the exact formulas for the finite temperature contribution in Eq. (11), not just the high temperature expansion of Eq. (14).

If the reheating temperature $T_{R}$ of the Universe is above the soft masses of all the squarks, a FOPT may take place. In this scenario, at high temperature, the effective potential has only one minimum at $\phi_{d}=0$, and the field $\tilde{d}_{R}$ starts off its postreheating evolution at the origin. As the temperature decreases, a nontrivial vacuum structure emerges; the effective potential develops a new minimum at $\phi_{d} \sim T$, and the Universe can undergo a FOPT from $\phi_{d}=0$ to this newly developed vacuum. Bubbles of true vacuum are nucleated, expand, and eventually fill out the entire Universe. During this process, GWs are emitted from sound shock waves, bubble collisions, and magnetohydrodynamic turbulence in the plasma. The frequency of emitted GWs is determined by the scale $M_{\tilde{q}}$. When the temperature drops below the squark masses, the vacuum situated away from the origin disappears, and the Universe evolves to the electroweak vacuum.

The resulting GW spectrum depends on the shape of the effective potential. It is determined by the Euclidean action for the saddle point configuration interpolating between the false and true vacua [51]. The spectrum is described by four parameters: the bubble wall velocity $v_{w}$ (we assume $\left.v_{w}=0.6 c\right)$, the nucleation temperature $T_{*}$, the strength of the FOPT $\alpha$, and the duration of the FOPT $1 / \tilde{\beta}$. Upon finding the Euclidean action corresponding to the nucleation temperature, we determined the $\mathrm{GW}$ parameters $\alpha$ and $\tilde{\beta}$ using standard formulas [52]. The dominant contribution to the GW signal comes from sound waves and is given by ${ }^{1}[56,57]$

$$
\begin{aligned}
h^{2} \Omega_{s}(\nu) \approx & \left(1.86 \times 10^{-5}\right) \frac{v_{w}}{\tilde{\beta}}\left(\frac{\kappa_{s} \alpha}{1+\alpha}\right)^{2}\left(\frac{100}{g_{*}}\right)^{\frac{1}{3}} \\
& \times \frac{\left(\frac{\nu}{\nu_{s}}\right)^{3}}{\left[1+0.75\left(\frac{\nu}{\nu_{s}}\right)^{2}\right]^{\frac{7}{2}}},
\end{aligned}
$$

where the parameter $\kappa_{s}$ is [58]

\footnotetext{
${ }^{1}$ It has recently been argued that the amplitude of the GW signal from sound waves includes an additional suppression factor [53]. However, given the large uncertainties in FOPT calculations [54,55], Eq. (20) serves as an accurate order of magnitude estimate of the expected signal strength.
} 


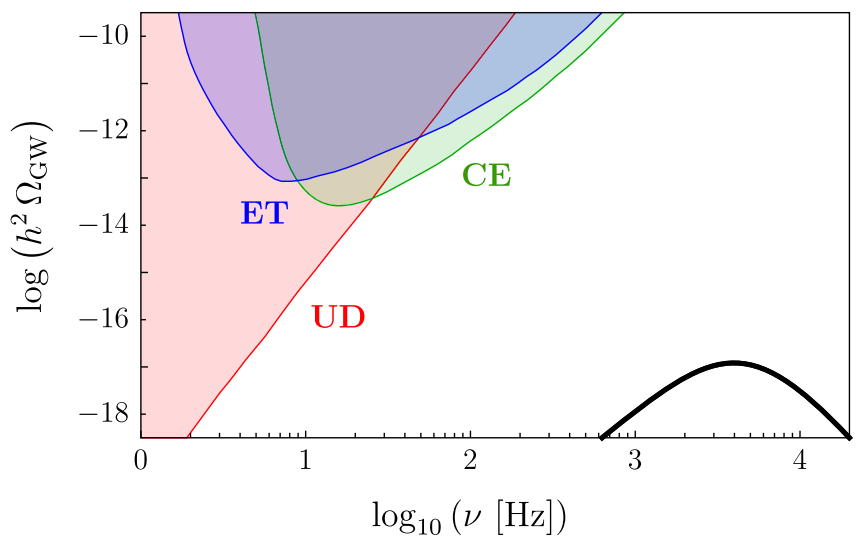

FIG. 2. Gravitational wave signature (black line) resulting from a first order phase transition in the minisplit SUSY for $T_{R}>M_{\tilde{q}}=10 \mathrm{PeV}, m_{\tilde{d}}=1 \mathrm{PeV}$, and a nucleation temperature $T_{*} \approx 4.9 \mathrm{PeV}$. Overplotted are the predicted sensitivities of the GW detectors: Einstein Telescope [59] (blue), Cosmic Explorer [3] (green), and U-DECIGO [60] (red).

$$
\kappa_{s} \approx \frac{6.9 v_{w}^{6 / 5} \alpha}{1.36-0.037 \sqrt{\alpha}+\alpha}
$$

and the peak frequency is

$$
\nu_{s}=(0.19 \mathrm{~Hz}) \frac{\tilde{\beta}}{v_{w}}\left(\frac{g_{*}}{100}\right)^{\frac{1}{6}}\left(\frac{T_{*}}{1 \mathrm{PeV}}\right) .
$$

Figure 2 shows the expected GW signal for the minisplit SUSY scenario considered (black line). Assuming the soft masses $M_{\tilde{q}}=10 \mathrm{PeV}$ and $m_{\tilde{q}}=1 \mathrm{PeV}$, the nucleation temperature of the FOPT is $T_{*} \approx 4.9 \mathrm{PeV}$. The signal peaks at frequencies $\sim \mathrm{kHz}$, but its maximal amplitude is only $h^{2} \Omega_{\mathrm{GW}} \sim 10^{-17}$, which is below the sensitivity of the near-future GW detectors: Cosmic Explorer, Einstein Telescope, and Ultimate DECIGO. This is due to the suppression of the strength parameter, which in this case can be estimated as $\alpha \sim \lambda v(T)^{4} /\left(\pi^{2} g_{\mathrm{eff}} T^{4} / 30\right)$, where $\lambda$ is the quartic coupling in Eq. (7), leading to a small $\alpha \sim 10^{-4}$.

\section{BEYOND THE MSSM}

Although the GW signal within the minimal MSSM is beyond the reach of upcoming experiments, it can be significantly enhanced if the MSSM particle spectrum is augmented by a large number of new families of squarks (so that there are $N$ copies of squarks in the theory, where $N \gg 1$ ). In this case, invoking a similar mass hierarchy between the squarks as before, i.e., all of the $N$ LH squarks and $\tilde{d}_{R}$ being much lighter than the remaining $(N-1) \mathrm{RH}$ squarks, the resulting constant in Eq. (17) becomes $C \ll-0.003$, leading to a deeper vacuum and an enhanced GW signal. Figure 3 shows the predicted GW signal (black line) for $N=30$ and the same choice of parameters as in
Fig. 2, which corresponds to a nucleation temperature $T_{*} \approx 1 \mathrm{PeV}$.

If two of the RH squarks, e.g., $\tilde{d}_{R}$ and $\tilde{s}_{R}$, are lighter than the other RH squarks and both develop vevs, an even richer vacuum structure emerges. The resulting effective potential exhibits an $\mathrm{SO}(2)$ symmetry and has a new minimum at $\phi_{d}^{2}+\phi_{s}^{2} \sim T^{2}$, which constitutes a circle in the $\left(\phi_{d}, \phi_{s}\right)$ field space. Similarly to the previous case, this new vacuum vanishes as the temperature becomes sufficiently small, leaving only one vacuum at $\phi_{d}=\phi_{s}=0$.

This setup is sufficient to induce a FOPT at the scale $M_{\tilde{q}}$, which results in GW signals (see Sec. V). In addition, some general features which naturally appear in the minisplit SUSY scenario may further lead to another FOPT at lower temperatures and the production of DWs. This is because the $\mathrm{SO}(2)$ degeneracy of the vacuum may be lifted-extra quartic terms for the squark fields breaking the $\mathrm{SO}(2)$ symmetry appear generically upon SUSY breaking. In particular, RH squarks couple to the Higgs field and $\mathrm{LH}$ squarks through $A$-terms, e.g., $A_{a b} H_{d} \tilde{Q}_{L a} \tilde{d}_{R b}$ where $a, b$ are flavor indices. Such couplings induce contributions to $\tilde{d}_{R}$ and $\tilde{s}_{R}$ quartic interactions. The size of such $A$-terms is determined by the SUSY-breaking mechanism. In this study, we treat the quartic couplings in those contributions as free parameters,

$$
V_{A}=\left(\lambda_{d}\left|\tilde{d}_{R}\right|^{4}+\lambda_{s}\left|\tilde{s}_{R}\right|^{4}\right) .
$$

In order to avoid large thermal corrections to the effective potential induced by those terms, we take $\lambda_{d}, \lambda_{s} \ll g_{s}^{2} / 6$, which makes such contributions much smaller than the ones generated by the $D$-term. The quartic terms in Eq. (23) reduce the symmetry from $\mathrm{SO}(2)$ to $Z_{4}$. This results in a vacuum structure with four equally deep minima, one in each quadrant of the $\left(\phi_{d}, \phi_{s}\right)$ plane.

With a four-fold degeneracy between the vacua, a FOPT between them would not happen. However, the MSSM offers additional Lagrangian terms breaking this degeneracy. One can introduce flavor-changing soft mass terms, which break the $Z_{4}$ symmetry to $Z_{2} \times Z_{2}$. In particular, the MSSM Lagrangian contains the term

$$
V_{F}=\tilde{m}_{d s}^{2} \tilde{d}_{R}^{\dagger} \tilde{s}_{R}+\text { H.c., }
$$

which breaks the degeneracy between the vacua and preserves only the $Z_{2} \times Z_{2}$ symmetry along two diagonal directions in the $\left(\phi_{d}, \phi_{s}\right)$ plane. Although such flavorchanging soft mass terms generically cause phenomenological problems for $\mathrm{TeV}$-scale SUSY by inducing sizable FCNCs, those terms are not problematic in the minisplit MSSM due to large squark masses.

Depending on the initial condition and the reheating temperature, two qualitatively different scenarios occur. First, the Universe may undergo a FOPT at the heavy RH squark mass scale $\sim M_{\tilde{q}}$, along with a subsequent 
production of DWs. Second, the Universe may undergo a FOPT at a scale $\ll M_{\tilde{q}}$. These are outlined as follows:

(i) If the reheating temperature of the universe is above the soft masses of all the squarks, the effective potential at this temperature has only one minimum at $\phi_{d}=\phi_{s}=0$; thus, the fields $\tilde{d}_{R}$ and $\tilde{s}_{R}$ start off their postreheating evolution at the origin. As the temperature decreases, a nontrivial vacuum structure emerges; i.e., the effective potential develops a new vacuum at $\phi_{d}^{2}+\phi_{s}^{2} \sim T^{2}$, as mentioned above. If the $A$-term induced quartic interaction in Eq. (23) is negligible and the temperature is much higher than the soft mass terms of light squarks, the vacuum is approximately degenerate along a circle. Therefore, the Universe undergoes a FOPT from $\phi_{d}=\phi_{s}=0$ to the newly developed vacuum at $\phi_{d}^{2}+\phi_{s}^{2} \sim T^{2}$. The frequency of emitted GWs is determined by the scale $M_{\tilde{q}}$. With temperature further decreasing, the degeneracy of the vacuum is broken. Two pairs of vacua are formed with different energy densities because of the nonzero flavor-breaking term in Eq. (24). This leads to the formation of DWs. The DWs separating nondegenerate vacua annihilate very quickly. On the other hand, the DWs separating degenerate vacua exist until the temperature drops below the squark masses, i.e., until the vacua situated away from the origin disappear. After that, the Universe evolves to the electroweak vacuum.

(ii) The other case is when the reheating temperature is below the mass scale of the heavy RH squarks $M_{\tilde{q}}$. The squark fields $\tilde{d}_{R}$ and $\tilde{s}_{R}$ start off with a universal misalignment as the initial condition. At the beginning of the postreheating era the universe may reside in the same metastable vacuum in all Hubble patches, and, with decreasing temperature, undergo a strong FOPT to the true vacuum. The frequency of the produced GWs depends on the scale of $\tilde{m}_{d s}^{2}$ and the $A$-term quartic couplings.

As discussed in Sec. IV, when the temperature drops to $\mathcal{O}(\mathrm{PeV})$, the $Z_{4}$ symmetry of the vacuum is spontaneously broken down to $Z_{2} \times Z_{2}$ via the term in Eq. (24), and DWs form around the boundaries of regions corresponding to different vacua. The DW dynamics is governed by the tension $\sigma \sim v(T)^{3}$ and the difference between the true and false vacua energy densities $\Delta \rho_{\text {vac }}$. In our case, $v(T) \sim T$; therefore, $\sigma \sim T^{3}$. The DWs remain stable as long as the tension force $p_{T}$ given by

$$
p_{T} \sim \frac{\sigma T^{2}}{M_{\mathrm{Pl}}} \sim \frac{T^{5}}{M_{\mathrm{Pl}}}
$$

where $M_{\mathrm{Pl}}$ is the Planck mass, is larger than the volume pressure $p_{V}$, which reads

$$
p_{V} \sim \Delta \rho_{\mathrm{vac}} \sim \tilde{m}_{d s}^{2} T^{2}
$$

Since in our case $\tilde{m}_{d s} \sim 1 \mathrm{PeV}$, the DWs annihilate immediately after forming. This annihilation is the source of a stochastic GW background. The peak frequency of the GW signal is [61]

$$
\nu_{\mathrm{DW}}^{\max } \sim(0.1 \mathrm{~Hz}) \frac{T_{A}}{\mathrm{PeV}}
$$

whereas the amplitude at this frequency is

$$
h^{2} \Omega_{\mathrm{DW}}^{\max } \sim 10^{-32}\left(\frac{\sigma^{2}}{T_{A}^{4} \times \mathrm{PeV}^{2}}\right) \sim 10^{-32}\left(\frac{T_{A}}{\mathrm{PeV}}\right)^{2} .
$$

Thus, the GW signal from DWs is too weak to be detected in the foreseeable future.

We now turn to the case when the reheating temperature is below the mass scale of the heavy RH squarks and assume that the Universe began its postreheating evolution in one of the two metastable vacua generated by the $A$-term quartic couplings and the flavor-breaking term. The scale of the FOPT is thus governed by the parameter $\tilde{m}_{d s}^{2}$. Within the minisplit SUSY framework, we choose $m_{\tilde{q}}^{2} \sim \tilde{m}_{d s}^{2} \sim O(0.1) \mathrm{PeV}^{2}$, which sets the nucleation temperature at $T_{*} \sim \mathcal{O}(500 \mathrm{TeV})$. The only other free parameters in the effective potential are $\lambda_{d}$ and $\lambda_{s}$. For various choices of these parameters, we calculated the Euclidean action at the nucleation temperature using the software ANYBUBBLE [62] and used it to compute the FOPT

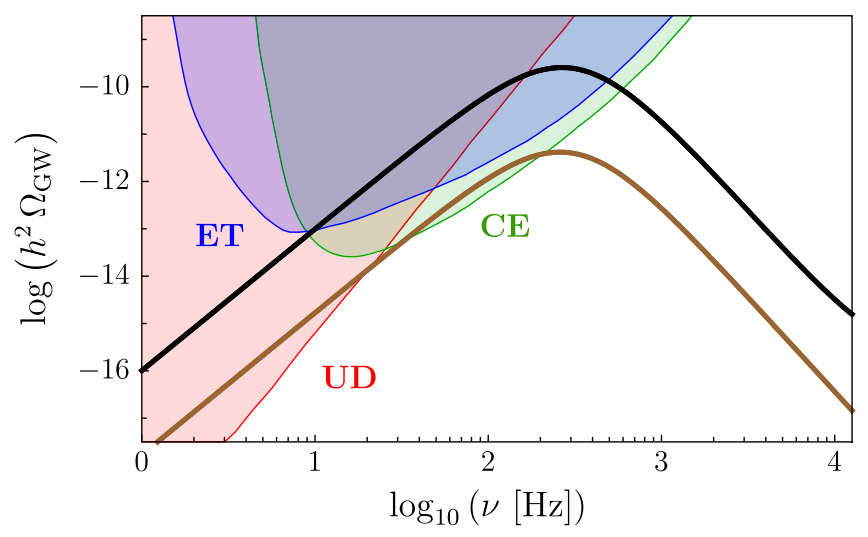

FIG. 3. Gravitational wave signatures of the MSSM extension with $N=30$ families of squarks. The black curve corresponds to a transition to a one-dimensional vacuum assuming the same parameters as in Fig. 2; the nucleation temperature is $T_{*} \approx 1 \mathrm{PeV}$. The brown curve corresponds to a transition between twodimensional vacua discussed in Sec. VI for $M_{\tilde{q}}=10 \mathrm{PeV}$ and $\tilde{m}_{d s}^{2}=0.1 \mathrm{PeV}^{2}$; the corresponding nucleation temperature is $T_{*} \approx 600 \mathrm{TeV}$. Overplotted are the predicted sensitivities of the GW detectors: Einstein Telescope [59] (blue), Cosmic Explorer [3] (green), and U-DECIGO [60] (red). 
parameters $\alpha$ and $\tilde{\beta}$, which were then plugged into Eq. (20) to derive the expected GW spectrum.

Figure 3 presents the GW signal (brown line) in the minisplit SUSY extended to $N=30$ families of squarks within scenario ii. The $A$-term quartic couplings were set to $\lambda_{d}=\lambda_{s}=0.03$, and the flavor-breaking term, along with the renormalization scale, was chosen to be $\tilde{m}_{d s}^{2}=\mu^{2}=0.1 \mathrm{PeV}^{2}$. This corresponds to a nucleation temperature $T_{*} \approx 600 \mathrm{TeV}$.

\section{CONCLUSIONS}

We demonstrated that within the framework of the minisplit supersymmetry it is possible for a phase with a reduced symmetry to develop at PeV-scale temperatures. The resulting finite temperature vacuum structure can be quite nontrivial, leading to a strong first order phase transition and/or the production of domain walls in the early Universe. The exact cosmological evolution depends on the assumptions regarding the reheating temperature. In the cases considered, a stochastic gravitational wave background at $\sim 1 \mathrm{kHz}$ frequencies is predicted. In the minimal formulation of the theory, the signal is not sufficiently strong to be detected at LIGO or the next generation of gravitational wave detectors.

Nevertheless, some extensions of the minisplit SUSY scenario may provide a much stronger gravitational wave signal. We showed that this is indeed the case when one introduces more scalars into the theory. It would be interesting to investigate which other modifications of the model lead to a nontrivial vacuum structure at finite temperature, while providing a measurable gravitational wave signal.

Finally, we note that our findings open the door to searches for a nontrivial vacuum structure of supersymmetric theories at high temperatures. Those efforts are complementary to the recently proposed gravitational wave search for supersymmetry-breaking hidden sectors [63].

\section{ACKNOWLEDGMENTS}

We are very grateful to the anonymous Physical Review D referee for extremely helpful comments regarding the manuscript. We also thank Andrei Angelescu, Peisi Huang, and Harikrishnan Ramani for useful discussions. The work of Y.Z. was supported in part by the U.S. Department of Energy under Award No. DE-SC0009959. J. H. Y. was supported by the National Natural Science Foundation of China (NSFC) under Grants No. 12022514, No. 11875003, and No. 12047503 and the National Key Research and Development Program of China Grant No. 2020YFC2201501. The work of B.S. is supported in part by the NSF Grant No. PHY-2014075.
[1] J. Aasi et al. (LIGO Scientific Collaboration), Advanced LIGO, Classical Quantum Gravity 32, 074001 (2015).

[2] M. Punturo et al., The Einstein Telescope: A third-generation gravitational wave observatory, Classical Quantum Gravity 27, 194002 (2010).

[3] D. Reitze et al., Cosmic Explorer: The U.S. contribution to gravitational-wave astronomy beyond LIGO, Bull. Am. Astron. Soc. 51, 035 (2019), https://baas.aas.org/pub/ 2020n7i035/release/1.

[4] S. Dimopoulos and H. Georgi, Softly broken supersymmetry and SU(5), Nucl. Phys. B193, 150 (1981).

[5] S. Dimopoulos, S. Raby, and F. Wilczek, Supersymmetry and the scale of unification, Phys. Rev. D 24, 1681 (1981).

[6] L. E. Ibanez and G. G. Ross, Low-energy predictions in supersymmetric grand unified theories, Phys. Lett. 105B, 439 (1981).

[7] W. J. Marciano and G. Senjanovic, Predictions of supersymmetric grand unified theories, Phys. Rev. D 25, 3092 (1982).

[8] L. Alvarez-Gaume, M. Claudson, and M. B. Wise, Lowenergy supersymmetry, Nucl. Phys. B207, 96 (1982).

[9] M. Carena, M. Quiros, and C. E. M. Wagner, Opening the window for electroweak baryogenesis, Phys. Lett. B 380, 81 (1996).
[10] M. Carena, M. Quiros, and C. E. M. Wagner, Electroweak baryogenesis and higgs and stop searches at LEP and the Tevatron, Nucl. Phys. B524, 3 (1998).

[11] B. de Carlos and J. R. Espinosa, The baryogenesis window in the MSSM, Nucl. Phys. B503, 24 (1997).

[12] J. M. Cline, G. D. Moore, and G. Servant, Was the electroweak phase transition preceded by a color broken phase? Phys. Rev. D 60, 105035 (1999).

[13] M. Carena, G. Nardini, M. Quiros, and C. E. M. Wagner, The baryogenesis window in the MSSM, Nucl. Phys. B812, 243 (2009).

[14] N. Blinov and D. E. Morrissey, Vacuum stability and the MSSM Higgs mass, J. High Energy Phys. 03 (2014) 106.

[15] W. G. Hollik, A new view on vacuum stability in the MSSM, J. High Energy Phys. 08 (2016) 126.

[16] N. Arkani-Hamed and S. Dimopoulos, Supersymmetric unification without low energy supersymmetry and signatures for fine-tuning at the LHC, J. High Energy Phys. 06 (2005) 073.

[17] N. Arkani-Hamed, S. Dimopoulos, G. F. Giudice, and A. Romanino, Aspects of split supersymmetry, Nucl. Phys. B709, 3 (2005).

[18] A. Arvanitaki, N. Craig, S. Dimopoulos, and G. Villadoro, Mini-split, J. High Energy Phys. 02 (2013) 126. 
[19] S. Weinberg, Gauge and global symmetries at high temperature, Phys. Rev. D 9, 3357 (1974).

[20] A. D. Linde, High density and high temperature symmetry behavior in gauge theories, Phys. Rev. D 14, 3345 (1976).

[21] R. N. Mohapatra and G. Senjanovic, Broken symmetries at high temperature, Phys. Rev. D 20, 3390 (1979).

[22] V. A. Kuzmin, M. E. Shaposhnikov, and I. I. Tkachev, Gauge hierarchies and unusual symmetry behavior at high temperatures, Phys. Lett. 105B, 159 (1981).

[23] V. A. Kuzmin, M. E. Shaposhnikov, and I. I. Tkachev, Baryon generation and unusual symmetry behaviour at high temperatures, Nucl. Phys. B196, 29 (1982).

[24] P. Salomonson, B. S. Skagerstam, and A. Stern, On the primordial monopole problem in grand unified theories, Phys. Lett. 151B, 243 (1985).

[25] Y. Fujimoto and S. Sakakibara, On symmetry non-restoration at high temperature, Phys. Lett. 151B, 260 (1985).

[26] G. Dvali, A. Melfo, and G. Senjanovic, Symmetry Nonrestoration at High Temperature and the Monopole Problem, Phys. Rev. Lett. 75, 4559 (1995).

[27] G. R. Dvali and G. Senjanovic, Is There a Domain Wall Problem? Phys. Rev. Lett. 74, 5178 (1995).

[28] J. Lee and I. Koh, Inflation and inverse symmetry breaking, Phys. Rev. D 54, 7153 (1996).

[29] N. Rius, Symmetry nonrestoration at high temperature, in International Workshop on Physics Beyond the Standard Model: From Theory to Experiment (Valencia 97) (World Scientific, Singapore, 1997), pp. 470-473 [arXiv:hep-ph/ 9801313].

[30] B. Bajc, High temperature symmetry nonrestoration, in 3rd International Conference on Particle Physics and the Early Universe (World Scientific, Singapore, 2000), pp. 247-253 [arXiv:hep-ph/0002187].

[31] J. R. Espinosa, M. Losada, and A. Riotto, Symmetry nonrestoration at high temperature in little Higgs models, Phys. Rev. D 72, 043520 (2005).

[32] M. Sakamoto and K. Takenaga, High temperature symmetry nonrestoration and inverse symmetry breaking on extra dimensions, Phys. Rev. D 80, 085016 (2009).

[33] P. Meade and H. Ramani, Unrestored Electroweak Symmetry, Phys. Rev. Lett. 122, 041802 (2019).

[34] I. Baldes and G. Servant, High scale electroweak phase transition: Baryogenesis \& symmetry non-restoration, J. High Energy Phys. 10 (2018) 053.

[35] A. Glioti, R. Rattazzi, and L. Vecchi, Electroweak baryogenesis above the electroweak scale, J. High Energy Phys. 04 (2019) 027.

[36] A. Angelescu and P. Huang, Strongly first order phase transitions from new fermions at the TeV scale, Phys. Rev. D 99, 055023 (2019).

[37] O. Matsedonskyi and G. Servant, High-temperature electroweak symmetry non-restoration from new fermions and implications for baryogenesis, J. High Energy Phys. 09 (2020) 012.

[38] B. Bajc, A. Lugo, and F. Sannino, The free and safe fate of symmetry non-restoration, Phys. Rev. D 103, 096014 (2021).

[39] H. E. Haber, Baryon asymmetry and the scale of supersymmetry breaking, Phys. Rev. D 26, 1317 (1982).
[40] M. L. Mangano, Global and gauge symmetries in finite temperature supersymmetric theories, Phys. Lett. 147B, 307 (1984).

[41] B. Bajc, A. Melfo, and G. Senjanovic, On supersymmetry at high temperature, Phys. Lett. B 387, 796 (1996).

[42] G. R. Dvali and L. M. Krauss, High temperature symmetry breaking, SUSY flat directions, and the monopole problem, arXiv:hep-ph/9811298.

[43] B. Bajc and G. Senjanovic, High temperature symmetry breaking via flat directions, Phys. Rev. D 61, 103506 (2000).

[44] A. Riotto and G. Senjanovic, Supersymmetry and Broken Symmetries at High Temperature, Phys. Rev. Lett. 79, 349 (1997).

[45] W. G. Hollik, G. Weiglein, and J. Wittbrodt, Impact of vacuum stability constraints on the phenomenology of supersymmetric models, J. High Energy Phys. 03 (2019) 109.

[46] M. Quiros, Field theory at finite temperature and phase transitions, Acta Phys. Pol. B 38, 3661 (2007), https://www .actaphys.uj.edu.pl/fulltext? series $=$ Reg $\&$ vol $=38 \&$ page $=3661$.

[47] D. Comelli and J. R. Espinosa, Bosonic thermal masses in supersymmetry, Phys. Rev. D 55, 6253 (1997).

[48] W.-C. Huang, F. Sannino, and Z.-W. Wang, Gravitational waves from Pati-Salam dynamics, Phys. Rev. D 102, 095025 (2020).

[49] C. Delaunay, C. Grojean, and J. D. Wells, Dynamics of nonrenormalizable electroweak symmetry breaking, J. High Energy Phys. 04 (2008) 029.

[50] D. Curtin, P. Meade, and H. Ramani, Thermal resummation and phase transitions, Eur. Phys. J. C 78, 787 (2018).

[51] A. D. Linde, Decay of the false vacuum at finite temperature, Nucl. Phys. B216, 421 (1983); Erratum, Nucl. Phys. B223, 544 (1983).

[52] J. Ellis, M. Lewicki, and J. M. No, On the maximal strength of a first-order electroweak phase transition and its gravitational wave signal, J. Cosmol. Astropart. Phys. 04 (2019) 003.

[53] H.-K. Guo, K. Sinha, D. Vagie, and G. White, Phase transitions in an expanding Universe: Stochastic gravitational waves in standard and non-standard histories, J. Cosmol. Astropart. Phys. 01 (2021) 001.

[54] D. Croon, O. Gould, P. Schicho, T. V. I. Tenkanen, and G. White, Theoretical uncertainties for cosmological first-order phase transitions, J. High Energy Phys. 04 (2021) 055.

[55] H.-K. Guo, K. Sinha, D. Vagie, and G. White, The benefits of diligence: How precise are predicted gravitational wave spectra in models with phase transitions? J. High Energy Phys. 06 (2021) 164.

[56] M. Hindmarsh, S. J. Huber, K. Rummukainen, and D. J. Weir, Gravitational Waves from the Sound of a First Order Phase Transition, Phys. Rev. Lett. 112, 041301 (2014).

[57] C. Caprini et al., Science with the space-based interferometer eLISA. II: Gravitational waves from cosmological phase transitions, J. Cosmol. Astropart. Phys. 04 (2016) 001.

[58] J. R. Espinosa, T. Konstandin, J. M. No, and G. Servant, Energy budget of cosmological first-order phase transitions, J. Cosmol. Astropart. Phys. 06 (2010) 028. 
[59] B. Sathyaprakash et al., Scientific objectives of Einstein Telescope, Classical Quantum Gravity 29, 124013 (2012); Erratum, Classical Quantum Gravity 30, 079501 (2013).

[60] S. Kuroyanagi, K. Nakayama, and J. Yokoyama, Prospects of determination of reheating temperature after inflation by DECIGO, Prog. Theor. Exp. Phys. (2015), 13E02.
[61] K. Saikawa, A review of gravitational waves from cosmic domain walls, Universe 3, 40 (2017).

[62] A. Masoumi, K. D. Olum, and B. Shlaer, Efficient numerical solution to vacuum decay with many fields, J. Cosmol. Astropart. Phys. 01 (2017) 051.

[63] N. Craig, N. Levi, A. Mariotti, and D. Redigolo, Ripples in spacetime from broken supersymmetry, J. High Energy Phys. 02 (2021) 184. 